\title{
Langerhans cell histiocytosis with multisystem involvement in an infant: A case report
}

\author{
LINTAO BI, BUTONG SUN, ZHENXIA LU, ZHANGZHEN SHI, DAN WANG and ZHENXING ZHU \\ Department of Hematology and Oncology, China-Japan Union Hospital of Jilin University, \\ Changchun, Jilin 130033, P.R. China
}

Received June 3, 2014; Accepted February 3, 2015

DOI: $10.3892 /$ etm.2015.2396

\begin{abstract}
Langerhans cell histiocytosis ( $\mathrm{LCH}$ ) is a proliferative disease of histiocyte-like cells, with a wide range of clinical presentations that vary from a solitary lesion to more severe multifocal or disseminated lesions. The disease can affect any age group; however, the peak incidence rate is in infants aged between 1 and 3 years-old. Diagnosis of LCH should be based on the synthetical analysis of clinical presentations, in addition to features of imaging and histopathology. Although certain cases regress spontaneously, other patients require systemic chemotherapy together with the administration of steroids. The present study reports the case of an infant with LDH with multisystem involvement, including that of the bone, skin, orbit, spleen and lungs. The patient received chemotherapy and obtained rapid improvement in the involved systems. A total of 2.5 years after completion of the therapy, the patient still remains in follow-up and no evidence of active disease has been noted.
\end{abstract}

\section{Introduction}

Langerhans cell histiocytosis ( $\mathrm{LCH}$ ), previously known as histiocytosis $\mathrm{X}$, is a proliferative disease of histiocyte-like cells. The condition is a rare disorder with several forms of clinical presentations, which include osteolysis, ulcerations of the skin and soft tissues, and occasionally viscera involvement (1). The majority of cases present as uni- or multifocal bone lesions, while visceral involvement is less common $(2,3)$. The most frequent sites of a visceral manifestation are the lungs, followed by the skin and the lymph nodes (2-5). The etiology of LCH is yet to be completely understood, although certain forms of immunological dysfunctions have been implicated. LCH can occur at any age; however, the peak incidence is observed in individuals aged between 1 and 3 years (6). A

Correspondence to: Dr Lintao Bi, Department of Hematology and Oncology, China-Japan Union Hospital of Jilin University, 126 Xiantai Street, Changchun, Jilin 130033, P.R. China

E-mail: haoya1968@gmail.com

Key words: Langerhans cell histiocytosis, multisystem involvement diagnosis of LCH should be based on the synthetic analysis of clinical presentations, as well as features of imaging and histopathology. Prognosis is dependent on a variety of factors, including the age of onset, the number of organs involved, the degree to which normal function of the organs is affected and the rate of disease progression $(7,8)$. For the majority of children with $\mathrm{LCH}$, the disease is self-resolving. However, for patients with multisystemic involvement, the most common treatment method is steroids and chemotherapeutic agents. The present study reports the case of an infant diagnosed with LCH with multisystem involvement, including that of the bone, skin, orbit, spleen and lungs.

\section{Case report}

Written informed consent was obtained from the patient's mother. An 11-month-old Chinese boy was admitted to the China-Japan Union Hospital of Jilin University (Changchun, China) following presentations of recurrent seborrheic dermatitis all over the skin, bilateral otorrhea and a mass on the scalp. The seborrheic dermatitis deteriorated, and ulcers developed on the skin of the bilateral groin. In addition, the patient had a high fever of up to $103^{\circ} \mathrm{F}$. A decreased willing to stand or cry was observed when the patient was standing, along with exophthalmos of both eyes, particularly in the left eye. Physical examination revealed a soft mass, measuring $1.5 \mathrm{~cm}$ in diameter, in the right parietal region. Furthermore, computed tomography (CT) scans demonstrated a round-shaped osteolytic lesion of the right parietal bone (Fig. 1) and erosion of the right scapula (Figs. 2-3). In addition, CT imaging revealed a soft tissue mass in the left posterior orbit (Figs. 4 and 5). Increased interstitial markings, with reticular patterns in the lungs (Fig. 6), and enlargement of spleen (Fig. 7) were also observed in the CT scans. A complete blood count from the patient revealed a hemoglobin level of $112 \mathrm{~g} / \mathrm{l}$, a white blood cell count of $6.1 \times 10^{9} / 1$ and a platelet count of $456 \times 10^{9} / 1$. Anaplastic lymphoma kinase, liver and kidney function tests (alanine aminotransferase, $14 \mathrm{U} / \mathrm{l}$; aspartate aminotransferase, $22 \mathrm{U} / \mathrm{l}$; creatinine $1.0 \mathrm{mg} / \mathrm{dl}$ ) were normal. A skin biopsy was performed and the results confirmed the diagnosis of LCH. Immunohistochemical staining produced the following results: CD1a, +++; fascin, +++; S-100, +++; Kp-1, sparsely +; phosphoglucomutase-1, -; $\mathrm{Ki}-67,>20 \%+$. 
The patient subsequently received a chemotherapy regimen consisting of vindesine, etoposide and prednisone (VEP regimen). Following one cycle of chemotherapy, the patient's temperature returned to normal, with rapid improvement observed with regard to the dermatitis and otorrhea. In addition, the mass on the scalp decreased in size. The VEP regimen was then alternated with a VCP regimen, consisting of vindesine, cyclophosphamide and prednisone. After 12 cycles (one cycle every $\sim 4$ weeks), the patient was shifted to vinblastine therapy, which was applied every two weeks for one year, due to the major residual tumor burden. With completion of the therapy for $\sim 2.5$ years, the patient continues to undergo follow-up and no evidence of active disease has been observed.

\section{Discussion}

$\mathrm{LCH}$ is a rare disorder associated with a wide spectrum of presenting symptoms and variable clinical behavior and prognosis. Previously, the disease was separated into three classifications, including eosinophilic granuloma, Hand-Schüller-Christian and Letterer-Siwe (9). In 1990, a stratification system was adopted by the LCH Study Group that classified the disease into single system LCH and multisystem LCH (MS-LCH) (10). The former can be further divided into single site (unifocal bone, skin or lymph node) and multiple site (multifocal bone or lymph nodes) forms. MS-LCH is defined as having more than two organ systems involved at the time of diagnosis. In addition, MS-LCH can be subdivided into low-risk and high-risk forms. Low-risk patients have no involvement of high-risk organs, including the liver, lungs, spleen and hematopoietic system (11). However, high-risk patients have one or more of these organs involved (12). In the case of the present study, the patient presented with lungs and spleen involvement; thus, was classified in the high-risk group.

The diagnosis of LCH is based on histopathological examination of the biopsy specimens. Pathologically, LCH is characterized by multinucleated Langerhans' cells, histiocytes and eosinophils, although the hallmark cell is the Langerhans cell histiocyte. The gold standard for the diagnosis of the disease requires the presence of Birbeck granules on electron microscopy examination (13); however, in certain cases, immunohistochemistry can play a fundamental role in establishing a diagnosis. For example, a diagnosis can be established with the use of CD1a, S100 and/or CD45 immunostaining on histopathological specimens (14). Previously, a highly specific and sensitive monoclonal antibody against CD207 (langerin) has become commercially available. This protein appears to be important for the formation of the Birbeck-Broadbent granules (15-17). In the present study, a final diagnosis of LCH was confirmed based on the histopathological observations and immunohistochemical staining of the lesion cells with CD1a and S-100.

The etiology of $\mathrm{LCH}$, which is characterized by a clonal proliferation of histiocyte-like cells, is controversial. There remains uncertainty regarding whether $\mathrm{LCH}$ is a neoplastic or inflammatory disorder. $\mathrm{LCH}$ has been described as a neoplastic process due to the monoclonal proliferation of Langerhans cells (18). However, there is also the possibility that $\mathrm{LCH}$ is a reactive inflammatory disorder resulting from a dysregulation of the immune system (18). Previously, a

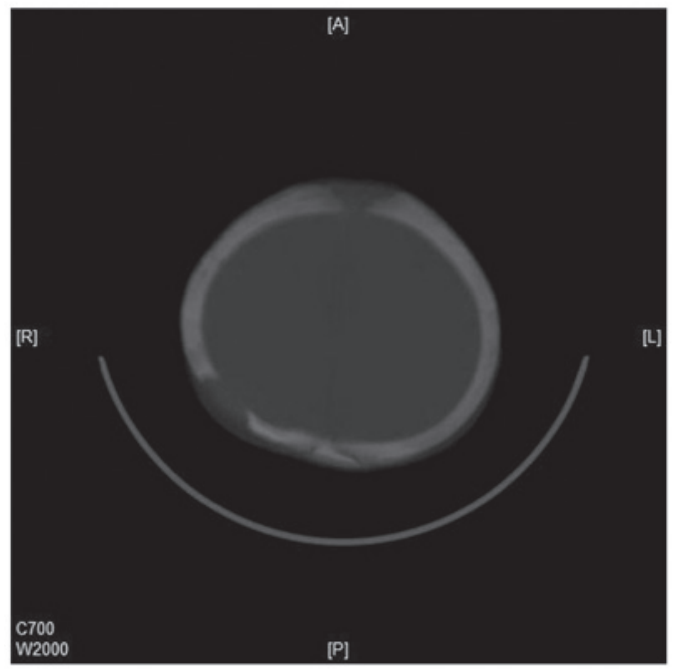

Figure 1. Computed tomography scan demonstrating a round-shaped osteolytic lesion of the right parietal bone.

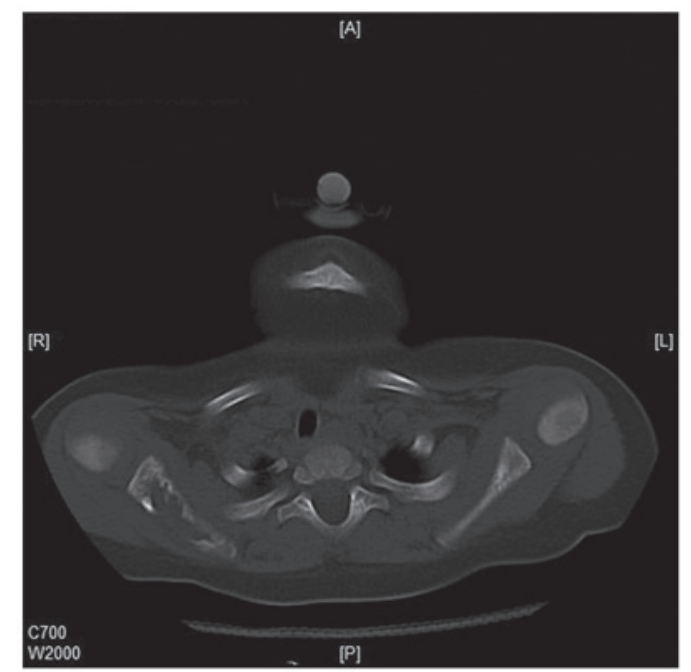

Figure 2. Computed tomography image showing osteolytic erosion of the right scapula.

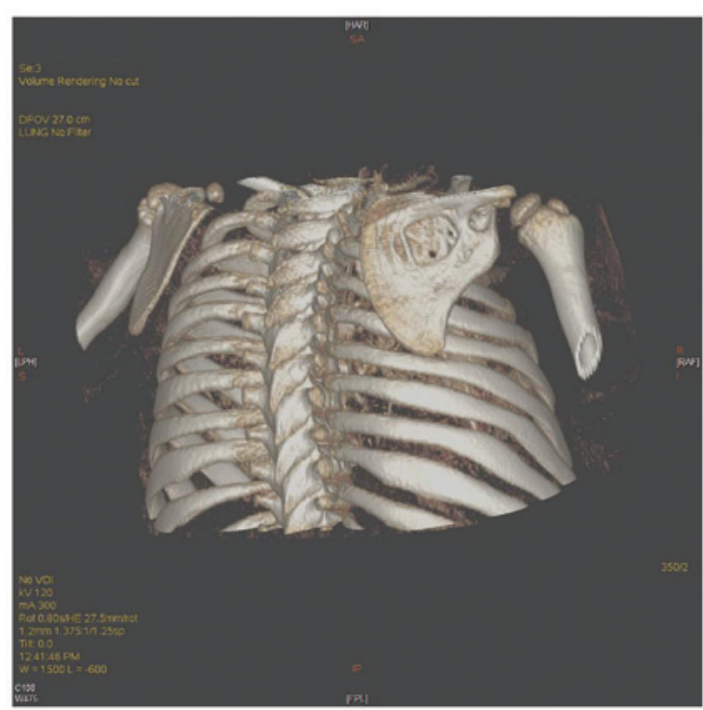

Figure 3. A three-dimensional computed tomography image showing the osteolytic erosion of the right scapula. 


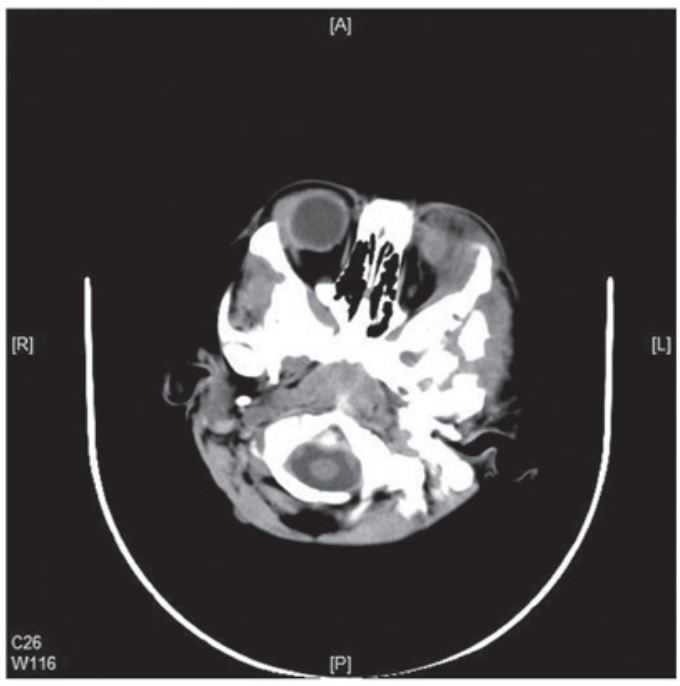

Figure 4. Computed tomography scan demonstrating the soft tissue mass in the left posterior orbit.

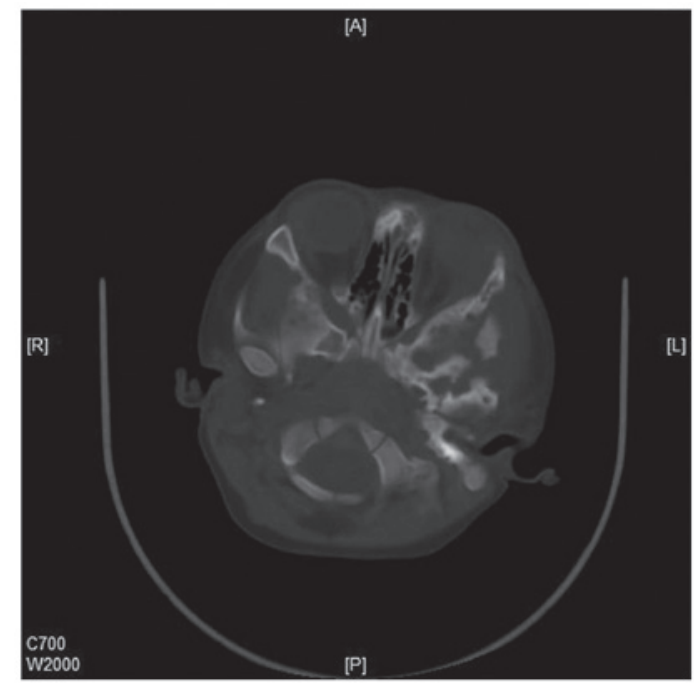

Figure 5. Computed tomography scan demonstrating the soft tissue mass in the left posterior orbit with bone erosion.

number of findings with regard to the molecular aspects of the disease suggested the possibility that LCH may be the result of an immune dysregulation (10,19-21). For example, Langerhans cell histiocytes are known to release a number of inflammatory chemokines, including $\mathrm{C}-\mathrm{C}$ chemokine receptor (CCR)1, CCR2, CCR5, CCR6, C-X-C chemokine receptor (CXCR)1, CXCR4, CCR7, chemokine (C-C motif) ligand (CCL)20/macrophage inflammatory protein (MIP)-3 $\alpha$, CCL2/ monocyte chemoattractant protein (MCP)-1, CCL3/MIP-1 $\alpha$, CCL4/MCP-4 $\alpha$, CCL5/RANTES, chemokine (C-X-C motif) ligand (CXCL8/interleukin (IL)-8 and CXCL10/IL-10. These chemokines are important for the recruitment of circulating immature dendritic cells, as well other immune cell types, such as T lymphocytes, macrophages and eosinophils. Subsequently, the recruited cells produce a number of additional cytokines, including IL-1, IL-3, IL-4, IL-5, IL-8, IL-10, granulocyte-macrophage colony-stimulating factor, tumor necrosis factor- $\alpha$, transforming growth factor- $\beta$

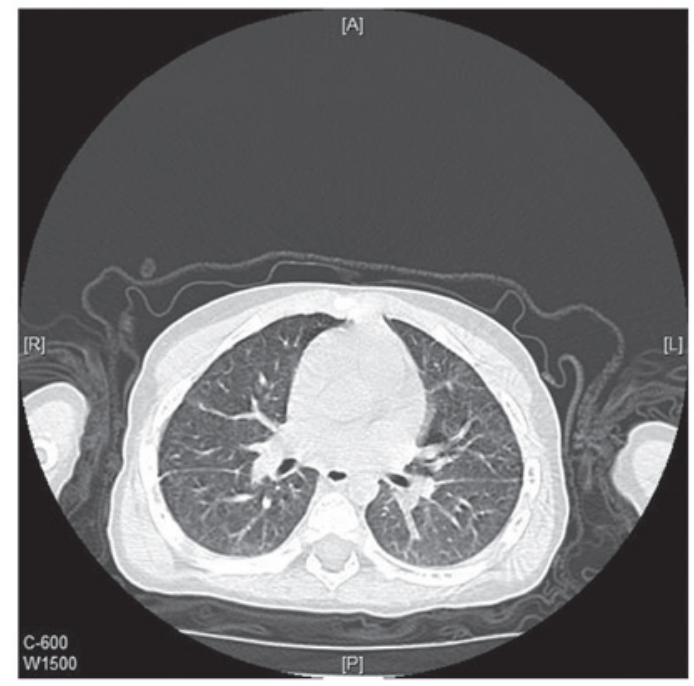

Figure 6. Computed tomography scan revealing increased interstitial markings in the lungs, with a number of markings showing a reticular pattern.

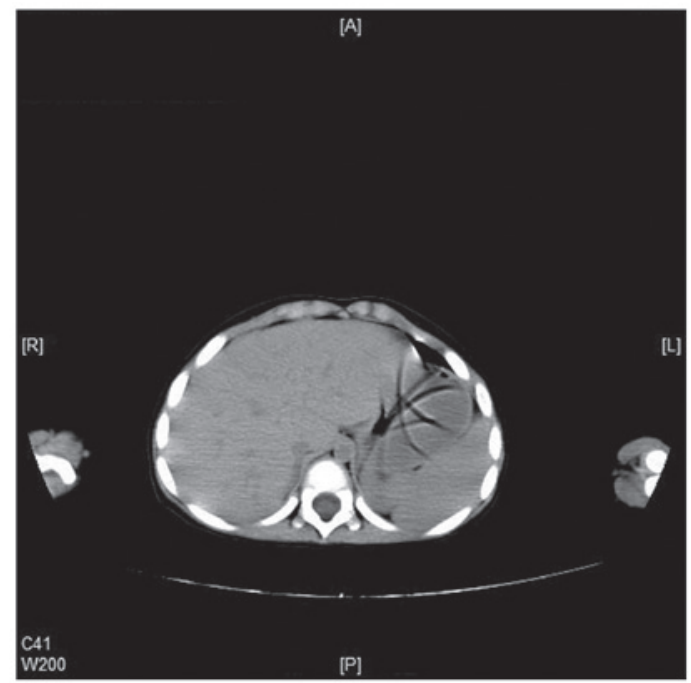

Figure 7. Computed tomography scan revealing the enlargement of the spleen.

and leukemia inhibitory factor. This particular immunological setting, known as a 'cytokine storm', in which autocrine and paracrine stimulation is established among the cells, can explain a number of clinical symptoms, including fever and failure to thrive (22). By contrast, numerous genetic abnormalities, including the loss of heterozygosity, damage to the chromosomes and damage to genes (such as BRAF mutation), have been detected in $\mathrm{LCH}$, which supports the possible clonal-expansive nature of the disease (22). These two pathways are currently under investigation with the aim to identify possible targets for the molecular therapy of LCH $(10,14,15,19-21,23-27)$. One of the most exciting findings that may have therapeutical potential is the presence of the BRAF V600E mutation in LCH. Previous studies have demonstrated that BRAF V600E mutations are present in $50-60 \%$ of patients with $\mathrm{LCH}(27,28)$. The $\mathrm{LCH}$ patients with a BRAF V600E mutation respond to BRAF V600E inhibition. Haroche et al reported a treatment protocol using vemurafenib 
(a BRAF inhibitor) in three patients with multisystemic and refractory Erdheim-Chester disease who carried the BRAF V600E mutation; two patients also had skin or lymph node LCH involvement. For all the patients, vemurafenib treatment resulted in a substantial and rapid clinical and biological improvement (29). However, more clinical trials are required to optimize the risk/benefit ratio of BRAF V600E inhibition in children.

Currently, the treatment for $\mathrm{LCH}$ with multisystem involvement is controversial. For patients with a multisystem disease, systemic multiagent chemotherapy is recommended. The most common chemotherapeutic agents are vinblastine, prednisone, etoposide and methotrexate, applied in various combinations (30). To date, a vinblastine- or etoposide-based regimen is most common for the treatment of $\mathrm{LCH}$ (31). However, severe and refractory $\mathrm{LCH}$ patients, particularly those with a life-threatening disease, may benefit from other therapies, including monoclonal antibodies that target CD1a, CD207 or CD52, specific cytokine inhibitors, 2-chlorodeoxyadenosine, BRAF V600E inhibition and bone marrow transplantation (28-32). In the present study, the patient was found to have a multivisceral form of the disease; thus, the VEP and VCP regimens were selected and applied for a year, after which the patient received vincristine therapy every two weeks for a year.

$\mathrm{LCH}$ is a rare proliferative disease of histiocyte-like cells that most commonly affects individuals in childhood. Diagnosis of LCH should be based on the synthetical analysis of clinical presentations, in addition to features of imaging and histopathology. Although certain cases regress spontaneously, other cases require systemic chemotherapy. The present study reported a rare infant case of $\mathrm{LCH}$ presenting with multisystem involvement, including that of the bone, skin, orbit, spleen and lungs. A vindesine- and prednisone-based regimen was selected for treatment and the patient was shown to progress well. However, the patient continues to undergo close follow-up to assess for signs of recurrence.

\section{References}

1. Satter EK and High WA: Langerhans cell histiocytosis: A review of the current recommendations of the Histiocyte Society. Pediatr Dermatol 25: 291-295, 2008.

2. Favara BE, McCarthy RC and Mierau GW: Histiocytosis X. Hum Pathol 14: 663-676, 1983.

3. Lieberman PH, Jones CR, Steinman RM, et al: Langerhans cell (eosinophilic) granulomatosis. A clinicopathologic study encompassing 50 years. Am J Surg Pathol 20: 519-552, 1996.

4. Egeler RM and D'Angio GJ: Langerhans cell histiocytosis. J Pediatr 127: 1-11, 1995.

5. Schulze J, Kitz R, Gruttner HP, et al: Severe isolated pulmonary Langerhans cell histiocytosis in a 6-year-old girl. Eur J Pediatr 163: 320-322, 2004.

6. Lajolo C, Campisi G, Deli G, et al: Langerhans's cell histiocytosis in old subjects: Two rare case reports and review of the literature. Gerodontology 29: e1207-e1214, 2012.

7. Minkov M, Prosch H, Steiner M, et al: Langerhans cell histiocytosis in neonates. Pediatr Blood Cancer 45: 802-807, 2005.

8. McClain KL: Langerhans cell histiocytosis: what is the orphan telling us? Hematology (Am Soc Hematol Educ Program): 84-87, 2004.

9. Isaacs H Jr: Fetal and neonatal histiocytoses. Pediatr Blood Cancer 47: 123-129, 2006.
10. Abla O, Egeler RM and Weitzman S: Langerhans cell histiocytosis: Current concepts and treatments. Cancer Treat Rev 36: 354-359, 2010.

11. Allen CE and McClain KL: Langerhans cell histiocytosis: a review of past, current and future therapies. Drugs Today (Barc) 43: 627-643, 2007.

12. Lau EG, Stepenaskie S, Moran R, et al: 'Blueberry muffin' rash and large right thigh mass: a unique presentation of Langerhans cell histiocytosis. Dermatol Online J 19: 18568, 2013.

13. Herwig MC, Wojno T, Zhang $Q$ and Grossniklaus HE: Langerhans cell histiocytosis of the orbit: Five clinicopathologic cases and review of the literature. Surv Opthalmol 58: 330-340, 2013.

14. Kasper EM, Aguirre-Padilla DH, Alter RY, et al: Histiocytosis X: Characteristics, behavior and treatments as illustrated in a case series. Surg Neurol Int 2: 57, 2011.

15. Kairouz S, Hashash J, Kabbara W, et al: Dendritic cell neoplasms: an overview. Am J Hematol 82: 924-928, 2007.

16. Takahashi K, Harada M, Kimoto M and Kondo F: Diagnostic confirmation of Langerhans cell histiocytosis of the jaws with CD1a immunostaining: a case report. J Oral Maxillofac Surg 61: 118-122, 2003.

17. Valladeau J, Dezutter-Dambuyant C and Saeland S: Langerin/CD207 sheds light on formation of birbeck granules and their possible function in Langerhans cells. Immunol Res 28: 93-107, 2003.

18. Rizzo FM, Cives M, Simone V, et al: New insights into the molecular pathogenesis of langerhans cell histiocytosis. Oncologist 19: 151-163, 2014

19. Egeler RM, van Halteren AG, Hogendoorn PC, et al: Langerhans cell histiocytosis: fascinating dynamics of the dendritic cell-macrophage lineage. Immunol Rev 234: 213-232, 2010.

20. Allen CE, Li L, Peters TL, et al: Cell-specific gene expression in Langerhans cell histiocytosis lesions reveals a distinct profile compared with epidermal Langerhans cells. J Immunol 184: 4557-4567, 2010

21. Annels NE, Da Costa CE, Prins F, et al: Aberrant chemokine receptor expression and chemokine production by Langerhans cells underlies the pathogenesis of Langerhans cell histiocytosis. J Exp Med 197: 1385-1390, 2003.

22. Hicks J and Flaitz CM: Langerhans cell histiocytosis: current insights in a molecular age with emphasis on clinical oral and maxillofacial pathology practice. Oral Surg Oral Med Oral Pathol Oral Radiol Endod 100 (2 Suppl): 42-66, 2005.

23. Bechan GI, Egeler RM and Arceci RJ: Biology of Langerhans cells and Langerhans cell histiocytosis. Int Rev Cytol 254: 1-43, 2006.

24. De Filippi P, Badulli C, Cuccia M, et al: Specific polymorphisms of cytokine genes are associated with different risks to develop single-system or multi-system childhood Langerhans cell histiocytosis. Br J Haematol 132: 784-787, 2006.

25. Egeler RM, Annels NE and Hogendoorn PC: Langerhans cell histiocytosis: a pathologic combination of oncogenesis and immune dysregulation. Pediatr Blood Cancer 42: 401-403, 2004.

26. Chikwava KR, Hunt JL, Mantha GS, et al: Analysis of loss of heterozygosity in single-system and multisystem Langerhans cell histiocytosis. Pediatr Dev Pathol 10: 18-24, 2007.

27. Badalian-Very G, Vergilio JA, Degar BA, et al: Recurrent BRAF mutations in Langerhans cell histiocytosis. Blood 116: 1919-1923, 2010.

28. Sahm F, Capper D, Preusser M, et al: BRAFV600E mutant protein is expressed in cells of variable maturation in Langerhans cell histiocytosis. Blood 120: e28-e34, 2012.

29. Haroche J, Cohen-Aubart F, Emile JF, et al: Dramatic efficacy of vemurafenib in both multisystemic and refractory Erdheim-Chester disease and Langerhans cell histiocytosis harboring the BRAF V600E mutation. Blood 121: 1495-1500, 2013.

30. Minkov M: Multisystem Langerhans cell histiocytosis in children: current treatment and future directions. Paediatr Drugs 13: 75-86, 2011.

31. Behrman RE, Kliegman RM and Jenson HB (eds): Class 1 histiocytoses. In: Nelson Textbook of Pediatrics. 16th edition. W.B. Saunders, Philadelphia, PA, pp1570-1572, 2000.

32. Jordan MB, McClain KL, Yan X, Hicks J and Jaffe R: Anti-CD52 antibody, alemtuzumab, binds to Langerhans cells in Langerhans cell histiocytosis. Pediatr Blood Cancer 44: 251-254, 2005. 